and from the standard lamp. The two agree and give a flux of $3.50 \times 10^{-20} \mathrm{ergs} \mathrm{s}^{-1} \mathrm{~cm}^{-2} \mathrm{~Hz}^{-1}$ or $3.40 \times 10^{-9} \mathrm{ergs} \mathrm{s}^{-1} \mathrm{~cm}^{-2} \AA^{-1}$.

This figure is substantially lower than was adopted by Code (1960) or found by Willstrop (1965).

\title{
BIBLIOGRAPHY
}

Bahner, K. 1963, Ap. J., 138, 1314.

Code, A. D. $\quad 1960$, Stars and Stellar Systems, vol. VI, Stellar Atmospheres, Ed. J. L. Greenstein, University of Chicago Press, Chicago, p. 50.

Oke, J. B. 1964, Ap. J., 140, 689.

Willstrop, R. V. $\quad 1965$, Mem. R. astr. Soc., 69, 83.

Wolff, S. C., Kuhi, L. V., Hayes, D. 1968, Ap. J., 152, 871.

J. B. OKE

Chairman of the Working Group

\section{9b. WORKING GROUP ON LINE INTENSITY STANDARDS}

Systematic equivalent width comparisons as initiated by $\mathrm{K}$. $\mathrm{O}$. Wright during the Seventh General Assembly of the IAU in 1948 and continued by G. Cayrel de Strobel since the Twelfth General Assembly in 1964 are meeting less and less response from the astronomical community. Griffin has stressed systematic errors in spectrographic results. For instance he claims in his paper (M.N.R.A.S., $143,319)$ that owing to the light thrown in the wings of the instrumental profiles, the observed equivalent widths of absorption lines in late type stellar spectra are 5 to $10 \%$ less than the true values. Furthermore Griffin pointed out the weakness of purely spectrographic comparisons which are all affected by the same type of errors. What is really needed is to know the true equivalent width of a few lines as they can be obtained from an accurate double pass photoelectric scanner. Pagel suggested that this can be done by comparing spectra of integrated sunlight from sky or minor planets with scans obtained by solar spectrometers. Griffin prefers that a bright star be used for this purpose. A complete change in the activity of this working group could very well be decided if the primary object is to have true standards of equivalent widths and if these cannot be obtained from conventional high dispersion spectrography.

G. CAYREL DE STROBEL

Chairman of the Working Group

29c. GROUPE DE TRAVAIL SUR LES ÈTOILES Be

\section{Spectrophotométrie, variations, vitesses radiales}

A. Peton (Meudon) à partir de spectres à petite dispersion, a évalué une période de 33 ans pour les variations de l'émission de HD4180, une classe B5 IV et des oscillations assez importantes de l'enveloppe absorbante sur 3-4 ans (non publ.).

F. R. Hickok (Dunlap) a étudié les structures fines et les vitesses radiales de $\phi$ Per à grande dispersion de telle sorte que les courbes de vitesse sont plus détaillées, mais, la période ne change pas. Probablement binaire. Des difficultés sont soulignées (non publ.).

Des variations spectrales et de vitesse radiale ont été observées par M. Hack et P. Stenner (Trieste, dans l'enveloppe de $\zeta$ Tau (publ.) ainsi que par Van der Wel (Utrecht) pour la période 1964-66 (non publ.). A. M. Delplace (Meudon) a mis en évidence une relation très étroite entre les profils Balmer $H$ et la période de 7 ans. L'enveloppe, en moyenne, est en récession (publ.).

J. P. Swings (Liège), N. L. Burnichon, D. Chalonge et L. Divan (I.A. Paris) ont déterminé le spectre continu et la classe spectrale de l'étoile HD45677 (B2 IVe) ainsi que l'identification des 
raies spectrales dans l'ultraviolet (publ.). Grâce à l'étude d'une longue série de spectres, une étude de l'évolution de cette étoile est en cours par J. P. Swings.

A. Peton a étudié de nombreux clichés à grande dispersion de HD45910 et de HD218393. Les points communs sont, essentiellement, le dédoublement des termes élevés de la série de Balmer et la disparition de l'enveloppe métallique en absorption alors que l'émission des raies métalliques est très forte (non publ.).

Une courte Note sur une nouvelle explosion de l'enveloppe de $48 \mathrm{Lib}$ a été soulignée par A. B. Underhill et H. G. Geuverink (Utrecht) (publ.) alors que Geuverink a fait une comparaison étendue des spectres des années 1957-58 et 1967-68 (non publ.) et que R. Faraggiana (Trieste) a étudié les raies les plus intenses dans le jaune-rouge dans les spectres des années 1950 à 1962, pris à Victoria par A. B. Underhill (publ.).

Mart de Groot (Utrecht) a mis en évidence, sur des spectres de PCyg, à grande dispersion, pris de 1942 à 1964, plusieurs couches dans l'enveloppe en expansion. Période de vitesse radiale de la couche externe: $114 j$. Des pulsations peuvent rendre compte de certains phénomènes (publ.). Dans son étude sur la perte de masse dans les supergéantes jeunes, Hutchings (Victoria) a étudié particulièrement PCyg (sous presse).

Spectrophotométrie de certaines raies de $\beta$ Lyr par S. N. Svolopoupos (publ.) L. Houziaux et Y. Andrillat (Mons et Montpellier) ont trouvé une température de $9750^{\circ} \pm 300 \mathrm{~K}$ pour l'enveloppe de HD217050 par comparaison des séries de Paschen et de Balmer de $\mathbf{H}$ (publ.).

M. et C. Jaschek et S. Malaroda ( La Plata) décrivent les spectres de plusieurs Be et signalent que des composantes d'émission à $\mathrm{Her}$ semblent plus fréquentes qu'on ne le pensait précédemment (publ.). C. Jaschek suit un certain nombre de Be Sud régulièrement pour surveiller leurs changements spectraux. W. Buscombe (Evanston) a photographié un certain nombre de spectres de Be Sud à grande dispersion, ce qui est rare. Les spectres à petite dispersion seront donnés prochainement. Il a obtenu également des spectres de Be Nord ressemblant aux Be Sud étudiées par lui.

D. Briot (Paris) a calculé les décréments Balmer pour 56 étoiles de B0 à B9 pour une corrélation entre type spectral et décrément.

K. Kadaira et M. Scholz (Mt. Wilson et Palomar) ont observé une diminution de l'émission $\mathbf{H}$ dans le spectre de HD58343 en 1968-69 par rapport à 1967-68. Les continus Paschen et Balmer sont toujours proportionnels à $y^{-3}$. En janvier 1968, $\mathrm{H} \alpha / \mathrm{H} \beta$ était égal à 2-3. Ils discutent les diverses théories concernant les enveloppes Be et concluent en pensant que cette étoile est une "shell" (non publ.).

S. Malaroda et Levato ( $L$ a Plata) mesurent actuellement les vitesses de rotation d'un certain nombre de Be Sud alors que W. Buscombe a fait un travail sur ce même sujet et a montré que 2 ou 3 étoiles sont proches du "break-up", ce qui pourrait obtenir des phénomènes intéressants à observer (publ.).

J. B. Hutchings a pu montrer que les étoiles chaudes (en absorption ou émission) ont des profils de raies extrèmement variables avec le temps. Le groupe de Meudon (Delplace, Duval, Herman, Peton) a trouvé des variations de vitesse radiale dans les enveloppes de $\zeta$ Tau et de HD45910, indiquant l'hétérogénéité de l'enveloppe, en particulier, au moment d'une expansion interne (publ.) et a montré également que le profil de la raie $\mathrm{H} \beta$ de HD 109387 varie rapidement avec une période probable de $1 \mathrm{~h} 10 \mathrm{~min}$, confirmée par la photoélectricité (publ.).

\section{Photométrie photoélectrique}

A. Feinstein (La Plata) a continué les observations en $U B V R I$ pour les Be Sud (publ.). Ces nouvelles mesures vérifient que presque toutes ces étoiles sont variables. C'est également le résultat du travail de C. Jaschek et Lia Ferrer. A. Feinstein veut mesurer $\mathrm{H} \alpha, \mathrm{H} \beta$ et $\mathbf{H} \gamma$ à l'aide de filtres interférentiels étroits et larges pour chaque raie.

Th. Schmidt-Kaler (Bochum) a fait la photométrie $U B V$ de 200 naines Be à l'aide du télescope photométrique de $61 \mathrm{~cm}$ du Chili (E.S.O.).

J. H. Bigay, R. Garnier et G. Adam (Lyon) à l'aide d'un dispositif comprenant 3 filtres interféren- 
tiels étroits: fil. $4740(100 \AA)+$ fil. $\mathbf{H} \beta(35 \AA)+$ fil. $5000(100 \AA)$, ont pu observer des variations rapides de magnitude de $\mathrm{H} \beta$, concommittantes à celles observées par spectrophotométrie à grande dispersion pour HD109387. D'autres mesures faites en $U B V$ sur HD 109387,45910 et 23642 sont en cours.

De façon tout à fait différente N. Morguleff et M. P. Veron (I.A. Paris) ont utilisé 8 bandes choisies dans le spectre (spectro. Barbier) et ont pu classer 60 Be Nord. Dans l'ensemble, les résultats sont en accord avec la classification Rojas-Herman.

\section{Théorie des Atmosphères}

T. Kogure (Mito) a déterminé les largeurs de raies d'émission de HD202904 qu'il considère comme une Be normale (publ.). L'étude concernant les profils de raies d'étoiles Be "pole-on" a été publié. Il a ensuite étudié la formation des raies Balmer dans les atmosphères étendues en considérant les comportements différents pour $n \leqslant 10,10<n \leqslant 20$ et $n<20$ dans la série de Balmer. Il explique ainsi les caractères Be et "shell" (publ.). En collaboration avec Duval et S. Itoh (Kyoto), il prépare la publication des diagrammes théoriques $(\mathrm{W} \alpha-\mathrm{H} \alpha / \mathrm{H} \beta$ calculés sur la base d'une atmosphère statique et qui permettront d'en déduire les propriétés cinématiques des atmosphères étendues.

D. N. Limber et J. M. Malborough (Yerkes) ont réalisé une nouvelle approche en ce qui concerne les forces pouvant entrer en considération pour le support des enveloppes d'étoiles Be (publ.) et Malborough a obtenu des profils de $\mathrm{H} \alpha$ en bon accord avec l'observation en utilisant 5 niveaux liés de $\mathrm{H}$ et une méthode d'approximation (publ.). Ces travaux vont continuer et, avec A. P. Cowley, ils vont comparer la théorie à l'observation. Un programme est déja écrit pour calculer les profils de raies dans le cas ou le taux de l'éjection est dépendante du temps.

\section{Catalogues ou cartes}

D.H. Menzel (Harvard) signale que tout le ciel sera bientôt fait en 3 couleurs (B, V, R) Nord et Sud.

L. R. Wackerling (Evanston): la liste de 5287 étoiles des premiers types à émission va être bientôt imprimé.

C. Jaschek (La Plata): le supplément au catalogue de Merrill-Burwell est prêt à être imprimé.

R. HERMAN

Président du Groupe de Travail 